Poster-discussion: Specific occupations and exposures 1

\title{
P56 TIME TO PREGNANCY STUDY IN MALE WORKERS EXPOSED TO VINYL CHLORIDE MONOMER IN THE POLYVINYL CHLORIDE INDUSTRY
}

Li-Ping Huang, ${ }^{1}$ Pei-Jen Chang, ${ }_{1}$ Ching-Chun Lin, ${ }^{2}$ Tsun-Jen Cheng, ${ }^{2}$ Pau-Chung Chen ${ }^{1}$ National Taipei University of Nursing and Health Sciences, Taipei, Taiwan; ${ }^{2}$ NTU, Taipei, Taiwan

10.1136/oemed-2011-100382.270

Objectives Although there were several published works on abnormal liver functions, liver cirrhosis or cancer, and genetic damages of the Taiwan polyvinyl chloride (PVC) cohort, the 
potential effect of vinyl chloride monomer (VCM) exposure on reproduction has not yet been studied.

Methods A retrospective time to pregnancy (TTP) study was conducted among the male workers and their spouses in three PVC factories in Taiwan. The detailed pregnancy history including time to pregnancy for each pregnancy and potential confounders was collected by face-to-face interview. We conducted the assessment of VCM job-exposure matrix using previous air-sampling data. Finally, we analysed the data of TTP using the discrete Cox's proportional hazards model. This study population consisted of 217 pregnancies in the analysis.

Results Compared to the non-exposure group, fecundability density ratio (FDR) were 0.87 (95\% CI: 0.58 to 1.32 ) and 0.60 (95\% CI: 0.34 to 1.05$)$ in low ( $<5 \mathrm{ppm})$ and high $(>=5 \mathrm{ppm})$ exposure groups, respectively.

Conclusions In summary, there might be a detrimental effect of VCM exposure on male fecundability. 